\title{
The Skyrmion Model and the Dynamical Breakdown of Chiral Symmetry
}

\author{
F. L. Braghint \\ Instituto de Física da Universidade de São Paulo \\ C.P. 66.318, C.E.P. 05315-970, São Paulo, SP, Brazil \\ I. P. Cavalcantei \\ Depto. de Física, CCET, Universidade Federal de Mato Grosso do Sul, \\ C.P. 549, C.E.P. 79070-900, Campo Grande, MS, Brazil
}

\begin{abstract}
A scalar field, $\eta(\mathbf{r})$, is coupled to the skyrmion. Its classical value in the vacuum ("condensate") reduces to the pion decay constant, $f_{\pi}$, being thus proportional to the chiral condensate $\langle\bar{q} q>$. A quadratic coupling of the scalar field to the pion kinetic Lagrangian term, reminiscent from the linear sigma model, is considered. Its mass is an additional free parameter in the model associated to the lightest scalar isoscalar meson. Solutions for the two resulting coupled differential equations are found in several cases. The nucleon, as a topological soliton, "digs a hole" in the vacuum making the value of the scalar condensate to be close to zero inside the skyrmion. Some of the observables, as for example the baryon masses, may have their values closer to the experimental ones. Derrick's stability analysis is applied to the model.
\end{abstract}

PACS numbers: 11.30.Qc/Rd; 12.38.Aw/Lg; 12.39.Dc/Fe; 12.40.Yx.

Key-words: Chiral condensate, chiral soliton, dynamical symmetry breaking, QCD, sigma meson, sigma models, topology, baryons.

IF-USP: 2000/2003.

\footnotetext{
*braghin@if.usp.br

†ipc@dfi.ufms.br
} 


\section{Introduction}

The description of processes involving strong interactions (mainly at low and intermediate energies) is very difficult in the frame of the Quantum Chromodynamics (QCD) due to its non abelian color and flavor structures and strong coupling constants. Thus effective models, like the Skyrme chiral soliton model (Skyrmion model), are constructed in such a way as to respect general properties from the more fundamental theory, such as chiral symmetry and its spontaneous breakdown [1].

The topological soliton model was formulated by Skyrme decades ago [2] possessing a conserved topological charge identified with the baryonic number. A deeper discussion about the real existence of minimum energy configurations in this model is found for example in [3]. The model encompasses chiral symmetry and its spontaneous breakdown and has further deeper motivation based on the large $N_{c}$ QCD analysis 4, 5]. The quantization of the rotational collective coordinates endows the soliton with spin half. It strengthens the interpretation of this soliton as a nucleon as long as it is possible to obtain predictions for hadronic properties [6]. An introductory study of the inclusion of quantum and thermal effects was done in [7].

Chiral spontaneous symmetry breaking is believed to be realized in the Nambu-Goldstone mode: the pion decay constant, $f_{\pi}$, is different from zero and the pion mass is small in the hadronic scale. $f_{\pi}$ can be seen as a "sigma condensate" of the linear sigma model. This sigma classical value is also identified to the chiral radius which is the constraint for the non linear realization of chiral symmetry as well. The non zero pion mass is usually assumed to break the chiral invariance of the Lagrangian explicitly, although only slightly. The vacuum is expected to be fulfilled with the scalar quark condensate $(<\bar{q} q>)$ which is proportional to the pion decay constant. To the lowest order, the Gell-Mann-Oakes-Renner relation, which relates QCD to hadronic degrees of freedom, reads:

$$
2<\bar{q} q>m_{q}=-f_{\pi}^{2} m_{\pi}^{2} .
$$

The experimental value of the pion decay constant is nearly $93 \mathrm{MeV}$. However, in the context of the Skyrmion model, it may reach very low values (such as $54 \mathrm{MeV}$ ) as long as one takes as input the physical values of nucleon and delta masses. This may suggest by itself that $f_{\pi}$ can acquire different values inside and outside the nucleon. The constant value of the chiral radius is one trivial (homogeneous) solution of the linear sigma model. We show in this work that there are other non trivial (non homogeneous) solutions in the presence of a nucleon, i.e., it occurs the formation of a "hole" in the vacuum due to the presence of the nucleon in the frame of the Skyrmion model. 
The coupling to QCD scalar degrees of freedom has already been envisaged in the frame of the Skyrmion model but in different approaches from that exploited here. Schechter and collaborators [8] and afterwards Waindzoch and Wambach [9] investigated such kind of effect by coupling a scalar field, representing a gluon degree of freedom. The value obtained for that "gluon condensate" inside the nucleon was much higher than in the vacuum, thus forming a kind of "bag". This strengthens some qualitative theoretical basis of the so called Bag Models [10]. In particular, for light glueballs, the bag was found to be very shallow [8]. These results are in agreement with the stabilizing mechanism discussed in [11. However the QCD vacuum is very complex and it is expected to contain, as discussed above, the quark-antiquark scalar condensate which is a manifestation of the spontaneously broken chiral symmetry. Thus a more detailed investigation of the contribution of this complex system for the structure of the nucleon is needed as well as the opposite, i.e., the effect of the nucleon on the vacuum. A more complex system was considered in [12] where a scalar meson field was also included.

We have earlier considered the chiral radius in the presence of hadrons as a dynamical variable [13, 14]. In this work we consider a dynamical scalar degree of freedom associated with the scalar chiral condensate. This is effectively implemented by the coupling of the Skyrmion to a self interacting scalar field. As shown is sections II and III, the scalar condensate ("sigma condensate") is constrained by the form of the potential which endows the model with the spontaneous symmetry breaking: in the vacuum it assumes the usual value of the pion decay constant, $\eta_{\text {vacuum }}=93 \mathrm{MeV}$. In the next sections we show the Lagrangian density and equations of the fields of this extended Skyrmion model. There are two coupled differential equations: for the soliton profile function, $F(r)$, and for the scalar isoscalar field, $\eta(r)$. The numerical solutions are investigated for some cases and hadronic observables are calculated. We investigate the effect of the magnitude of the mass of this scalar field - the additional free parameter of our model - privileging values between $560 \mathrm{MeV}$ and $980 \mathrm{MeV}$, which correspond respectively to the sigma (eventually the chiral partner of the pion) 115, 16, 17] and to the $f_{0}(980)$ meson [18]. The latter is one of the lightest well known scalar isoscalar mesons. The analysis of stability of the equations by means of dimensional arguments is also discussed in the section IV (Derrick's analysis). In section V we present our final comments, also discussing some ways of improving and extending the model. 


\section{$2 \quad$ Scalar field coupling}

We do not describe the Skyrmion model at length since this is done extensively in the literature. One natural way of imposing a coupling of a scalar field (such as the chiral partner of the pion) to the skyrmion model is to consider the linear realization of the sigma model. The degree of freedom of the sigma may be replaced by a variable (dynamical) chiral radius, $\eta(r)$, which becomes the new dynamical degree of freedom:

$$
f_{\pi} \rightarrow \eta=\sqrt{\pi^{2}+\sigma^{2}}=v+\epsilon,
$$

where $v=f_{\pi}$ is the classical value of the scalar field and $\epsilon$ its fluctuation [19. However, the resulting coupled model (roughly the linear sigma model) has no stable solitonic solution with non trivial topology as discussed later in this paper. Thus we include, as it is usually done in the standard Skyrmion model, the fourth order stabilizing term for the pionic sector. The modified Skyrmion Lagrangian density reads:

$$
\mathcal{L}=\frac{\eta(\mathbf{r})^{2}}{4} \operatorname{Tr}\left(\partial_{\mu} U^{\dagger} \partial^{\mu} U\right)+\frac{1}{32 e^{2}} \operatorname{Tr}\left(\left[U^{\dagger} \partial_{\mu} U, U^{\dagger} \partial_{\nu} U\right]^{2}\right)+\frac{1}{2} \mu^{2} f_{\pi} \eta(\mathbf{r})\left(\operatorname{Tr} U+\operatorname{Tr} U^{\dagger}-2\right)+\mathcal{L}_{\eta},
$$

where the first term is the Lagrangian density of the linear sigma model and the second one is introduced in order to stabilize the soliton [2]. While the first term becomes scale invariant with the quadratic coupling, the second one is scale invariant by itself and thus no such coupling has been

considered in this work for this term 1. $\mu^{2}$ is the pion mass whose Lagrangian term breaks slightly the chiral symmetry and scale invariance as well, at least in this form. $U$ is a chiral invariant matrix parametrized in terms of the profile function $F(\mathbf{r})$ within the Hedgehog ansatz (given by $\hat{\boldsymbol{\pi}}=\hat{\mathbf{r}}$ ):

$$
U(\mathbf{r})=\cos (F(\mathbf{r}))+i \boldsymbol{\tau} \cdot \hat{\boldsymbol{\pi}} \sin (F(\mathbf{r})) .
$$

Finally, the scalar field self interacting Lagrangian is chosen such that one recovers a classical value in the vacuum due to the (chiral) spontaneous symmetry breaking:

$$
\mathcal{L}_{\eta}=\frac{1}{2} \partial_{\mu} \eta(\mathbf{r}) \partial^{\mu} \eta(\mathbf{r})-\frac{1}{2} m_{\eta}^{2} \eta^{2}(\mathbf{r})-\frac{1}{4} \lambda \eta^{4}(\mathbf{r})
$$

The scalar field mass term breaks scale invariance but it is necessary for the consistency of the model. The bare scalar field mass parameter is $m_{\eta}^{2}$, being related to the physical scalar field mass $\left(m_{\epsilon}^{2}\right)$ by the following relation:

$$
m_{\eta}^{2}=\frac{1}{2}\left(\mu^{2}-m_{\epsilon}^{2}\right)
$$

\footnotetext{
${ }^{1}$ As a matter of fact, it is possible to introduce a scale invariant coupling in such term.
} 
The value of the condensate in the vacuum is found by the minimization of its potential. As stated above we consider two different possibilities for the physical particle associated with the scalar field. The $\eta$ field is expected to correspond to the lightest scalar-isoscalar meson. It may be a very broad scalar resonance in the s-wave of low energy $\pi-\pi$ scattering, with a mass in the range $500-800 \mathrm{MeV}$ that seems to have been observed in other processes [20, 16, 17]. In this case it would be associated to the chiral partner of the pion eventually corresponding to a Higgs field for Hadronic interactions $\mathrm{Q}$. We consider two different values for the scalar-isoscalar particle mass in order to investigate its effects on nucleon properties. The coupling constant $e$ may be related to the pion-pion scattering coupling as done in [22, 21], i.e., to a particular choice of the low energy constants (LECs) of some terms from the Chiral Perturbation Theory expansion [23]. In the works just quoted above the authors obtained: $e \simeq 4-7$. Large $N_{c}$ analysis with extrapolation to $N_{c}=3$ using sum rules of 24] leads to the approximated values: $e \simeq(7.6-12) / \sqrt{N_{c}}$. These values are, in principle, independent of $f_{\pi}$.

The coupling to the quadratic Lagrangian term of the Skyrmion model, as described above, is uniquely defined by the linear implementation of chiral symmetry. It is scale invariant. It is consistent with the consideration that the chiral radius is directly and unambiguosly related to the quarkantiquark condensate of QCD and, besides, to the pion decay constant, at least in the vacuum and at the tree level. For the coupling of the quartic stabilizing Skyrme term to the scalar field we found no scale invariant form.

Along this work we basically assume the chiral radius to be a dynamical variable, being equivalent to the sigma field. In this case one is led to the following replacement for the quadratic Skyrmion Lagrangian term:

$$
U(\mathbf{r}) \rightarrow \alpha(\mathbf{r}) U(\mathbf{r}), \quad \text { with } \quad \alpha(\mathbf{r})=\frac{\eta(\mathbf{r})}{f_{\pi}} .
$$

However, this replacement in the fourth order Skyrme term yields scale non-invariant Lagrangian terms and unstable solutions in the physical region for the parameters of the model. The lack of stability was also checked by the Derrick's argument [25] discussed in section IV.

Finally, one can notice that the fourth order Skyrme term may be derived from the coupling of the sigma model with the vector meson, $\vec{\rho}_{\mu}$, as a hidden or massive gauge boson [22, 26]. The above prescription for the function $U(\mathbf{r})$ may yield a $\eta(\mathbf{r})$-dependent $\rho$ mass and $e$ coupling such that scale invariance is mantained. This will be developed elsewhere. Although this is a very interesting idea

\footnotetext{
${ }^{2}$ Its origin, on the other hand, lies on the chiral spontaneous symmetry breaking of QCD vacuum.
} 
by itself, after the elimination of the $\rho$ degrees of freedom, the prescription seems to lead to other derivative and complicated terms which also may break scale invariance. At last it is interesting to preserve scale invariance in spite of the massive terms.

\section{Calculations}

In this section we investigate the solitonic solutions relative to equations of the fields of the model and afterwards their consequences for static nucleon observables. Adopting the usual hedgehog ansatz, $\hat{\boldsymbol{\pi}}=\hat{\mathbf{r}}$, the modified Lagrangian is written as:

$$
L=4 \pi \int d r r^{2}\left\{-\frac{\eta^{2}}{2} F^{2}-\frac{\sin ^{2}(F)}{e^{2} r^{2}} F^{\prime 2}-\frac{\sin ^{2}(F)}{r^{2}}-\frac{\sin ^{4}(F)}{2 e^{2} r^{4}}+\mu^{2} \eta f_{\pi} \cos (F)+\mathcal{L}_{\eta}\right\},
$$

where $F=F(r), \eta=\eta(r)$ and ' ${ }^{\prime}$ is the derivative with relation to the radial coordinate $r$.

The Euler-Lagrange equations for this system with the change of variable $r \rightarrow r f_{\pi}$ are given by:

$$
\begin{aligned}
& F^{\prime \prime}\left(r^{2} \eta^{2}+\frac{2 \sin ^{2}(F)}{e^{2}}\right)+2 r \eta^{2} F^{\prime}+\frac{\sin (2 F)}{e^{2}} F^{2}-\eta^{2} \sin (2 F)-\frac{4 \sin ^{3}(F) \cos (F)}{2 e^{2} r^{2}}\{+\} \\
& -\mu^{2} \eta \sin (F) r^{2} f_{\pi}+2 r \eta \eta^{\prime} F^{\prime}=0 \\
& \eta^{\prime \prime}+\frac{2}{r} \eta^{\prime}-\eta\left(F^{\prime 2}+\frac{2 \sin ^{2}(F)}{r^{2}}+m_{\eta}^{2}\right)+\mu^{2} \cos (F) f_{\pi}-\lambda \eta^{3}=0 .
\end{aligned}
$$

In the spatially homogeneous case of $\eta(r)=f_{\pi}$ (constant), the usual skyrmion equation and solutions are obtained for the first equation. That value for the sigma "condensate" (classical field) naturally emerges as a boundary solution in the limit $r \rightarrow \infty$, i.e., in the vacuum, far from the topological soliton for the solutions of interest. In the limit of $m_{\eta}^{2} \rightarrow \infty$ the equations become uncoupled, what is verified numerically.

For the scalar field equation we consider the following conditions:

$$
\begin{aligned}
& \eta(r \rightarrow \infty)=f_{\pi}, \\
& \eta^{\prime}(r \rightarrow \infty)=\eta^{\prime}(r=0)=0 .
\end{aligned}
$$

In this limit, in the vacuum, a constraint between the scalar field parameters is obtained. By minimizing the (homogeneous) scalar field potential from equation (8) we obtain:

$$
\lambda=\frac{\mu^{2}-m_{\eta}^{2}}{f_{\pi}^{2}}
$$


The corresponding solutions for the spatially non homogeneous case are presented later. Spontaneous chiral symmetry breaking occurs as long as

$$
\frac{\lambda}{m_{\eta}^{2}}<0
$$

which provides a condition for the existence of the "sigma condensate" at the classical level. Quantum fluctuations may considerably change this [27, 28]. They will be investigated elsewhere.

For the equation of the profile function $F(r)$ the usual boundary conditions still hold, i.e., winding number $n=1$, for which one considers $F(r=0)=\pi$ and $F(r \rightarrow \infty)=0$ [6]. From expression (8) one sees that it is possible to define an effective mass parameter for the field $\eta(r)$. Namely:

$$
m_{e f f}^{2}=m_{\eta}^{2}+F^{\prime 2}+\frac{2 \sin ^{2}(F)}{r^{2}} .
$$

Far from the nucleon this effective mass is just equal to $m_{\eta}^{2}$, but inside the skyrmion and close to it the contributions from the two other terms may be relevant.

\subsection{Numerical results}

We are thus faced with two differential equations with two point-type boundary conditions. They are solved in an iterative way by the "shooting" method using fourth order Runge Kutta with the boundary conditions shown above.

The numerical solutions for the skyrmion profile function are presented in figure 1 and, in figure 2, the solutions for the condensate $\eta(r)$. Three different cases are shown considering $f_{\pi}=93 \mathrm{MeV}$. The following values for the other input parameter were used for each solution:

$$
\begin{aligned}
& \text { (1) } m_{\epsilon}=980 \mathrm{MeV} \text { and } e=4, \\
& \text { (2) } m_{\epsilon}=980 \mathrm{MeV} \text { and } e=5, \\
& \text { (3) } m_{\epsilon}=560 \mathrm{MeV} \text { and } e=4 .
\end{aligned}
$$

From figure 1 we notice that the skyrmion radius becomes a little smaller and that inside the topological soliton the scalar field tends to decrease, i.e., the nucleon "digs a hole" in the vacuum otherwise fulfilled

with the condensate. For smaller (bigger) values of the scalar field mass the "hole" - which is in fact akin to the "bag" of other models - becomes deeper and wider (shallower and narrower). This is a mechanism for bag formation equal to those studied in [8, 9, 12]. As a matter of fact, the Skyrmion model and the constituent quark model were shown to yield equivalent results in the large $N_{c}$ limit [29], thus the bag structure should be expected in both models. 
In fact, we find that inside the topolotical soliton the effective mass given in expression (12) should be smaller in modulus, being even positive, thus violating condition (11) for the occurrence of spontaneous symmetry breaking. It can be seen as the restoration of the chiral symmetry inside the soliton (nucleon) for not high values of $m_{\eta}^{2}$. Therefore there is a sort of a "critical value" for the scalar particle mass above which the chiral symmetry is restored inside the nucleon. This value depends on the other parameters of the model and on the level of approximation for the quantum fluctuations of the fields.

Although it is not clear whether it is physically meaningful we describe the following curious issue. We have also investigated the possibility that at the origin, i.e., inside the nucleon, the classical scalar field is larger than in the vacuum. For a very particular fine tuned value of $\eta(r \rightarrow 0)-$ which depends on $e, m_{\eta}^{2}$ and $\eta(r \rightarrow 0)$ - we found a solution which goes asymptoticaly to $f_{\pi}$, as required and expected. We have obtained this only for large mass values. However this solution exhibits a sort of small amplitude oscillation, which decreases progressively with r, having large wavelength around the region of the topological soliton. It would correspond to a "stronger chiral symmetry breaking" inside the soliton. We leave the investigation of such solution for another work [30].

Strong and electromagnetic properties usually obtained for the skyrmion [6] were calculated considering the above scalar field coupling. The energy of the lightest baryons (nucleons) can be written as:

$$
\begin{aligned}
& M=4 \pi \int_{0}^{\infty} d r r^{2}\left\{\frac{\eta^{2}}{2}\left(F^{\prime 2}+\frac{2 \sin ^{2}(F)}{r^{2}}\right)+(1-\cos (F)) \mu^{2} \eta f_{\pi}+\frac{\eta^{2}}{2}+\frac{m_{\eta}^{2}}{2} \eta^{2}+\frac{\lambda}{4} \eta^{4}+\right. \\
& \left.+\frac{1}{e^{2}}\left(\frac{\sin ^{2}(F) F^{\prime 2}}{r^{2}}+\frac{\sin ^{4}(F)}{2 r^{4}}\right)+\frac{1}{\mathcal{I}}-E_{\text {vac }}\right\},
\end{aligned}
$$

where $\mathcal{I}$ is the moment of inertia [6] which now depends on $\eta(r)$ and $E_{v a c}$ is the vacuum energy due to the dynamical chiral symmetry breaking to be subtracted:

$$
E_{v a c}=\left(\frac{m_{\eta}^{2}}{4}-\frac{3 \mu^{2}}{4}\right) \text {. }
$$

In table 1 we compare the values of observables obtained for the usual Skyrmion model, our model (for the three cases shown in figures 1 and 2) and experimental data. A higher value for $e(e=5)$ yield better values for the mass spectrum as it is well known. However almost all the other observables (mainly the electromagnetic properties) still present values in disagreement with the experimental ones. On the other hand, considering smaller values of the scalar field mass, $m_{\epsilon}=560 \mathrm{MeV}$, a better agreement with the experimental values for most observables is obtained. The most remarkable 
exception is the behaviour of the axial coupling constant $g_{A}$. The $g_{A}$ coupling is calculated in two ways: 1) in the limit of zero momentum of the nucleon axial form factor and 2) by means of the Goldberger-Treiman relation [6] in which case all the parameters can be calculated from the resulting Skyrmion solutions. For $g_{A}$ and $g_{\pi N N}$ a smaller value of $e$ would be desirable, what would, in principle, make the values of the other observables to be in disagreement with experimental ones.

\section{$4 \quad$ Derrick's analysis of stability}

We have also performed the Derrick's analysis in order to verify the stability of the "finite sized" solutions of the differential equations [25]. It consists in checking, by dimensional arguments (scale transformations), whether there is a scale in which the total energy of the system is finite yielding stable finite size solutions for the corresponding equations. One has to consider scale transformations for the spatial coordinate variable

$$
r \rightarrow r^{\prime}=\frac{r}{\alpha}
$$

and for the dimensionful field

$$
\eta(r) \rightarrow \beta \eta\left(r^{\prime}\right)
$$

In fact it is more suitable to do it for the shifted equivalent field: $\theta(r)=f_{\pi}-\eta(r)$. Under the above transformations we verify whether the energy density has a stable minimum with relation to the scale transformation parameters, i.e.:

$$
\begin{array}{rlrl}
\frac{\delta M}{\delta \alpha} & =0, & & \frac{\delta M}{\delta \beta}=0, \\
\frac{\delta^{2} M}{\delta \alpha^{2}}>0, & \frac{\delta^{2} M}{\delta \beta^{2}}>0 .
\end{array}
$$

This analysis was performed for the following cases:

(i) Only the sigma model with the scalar field which has self interacting potential, i.e., no fourth order term for the pion field in the Lagrangian density (2);

(ii) The inclusion of the usual quartic Skyrme term exactly as it stands in expression (2);

(iii) The coupling of the scalar field in the fourth order Skyrme term, breaking the scale invariance, with the ansatz of expression (5). This modification yields, respectively, the following modified and additional Lagrangian density terms:

$$
\mathcal{L}_{4}=\frac{1}{f_{\pi}^{4} e^{2}}\left\{\eta^{4}(r)\left(\frac{s^{2} F^{\prime 2}}{r^{2}}+\frac{s^{4}}{2 r^{4}}\right)+\frac{\eta^{2} \eta^{\prime 2} s^{2}}{4 r^{2}}\right\} .
$$


In the first case (i) there are no stable solutions for the skyrmion profile function $\left(\delta^{2} M / \delta \alpha^{2}<0\right)$. It becomes clear once again the importance of the higher order derivative terms in order to keep the soliton against collapse, in spite of the problems with the convergence of the derivative series [31].

Case (ii) is stable provided that the chiral symmetry breaking term is small (as always assumed) compared to the self interacting scalar field term as it can be seen from the second equation of (19) below. The conditions for stability are given by:

$$
\begin{aligned}
& \frac{\delta^{2} M}{\delta \alpha^{2}}>0 \rightarrow \int_{0}^{\infty} r^{2} d r \frac{1}{e f_{\pi}}\left[2\left(2 \sin ^{2}(F) \frac{\left(F^{\prime}\right)^{2}}{r^{2}}+\frac{\sin ^{4}(F)}{r^{4}}\right)-\frac{\eta^{2}}{4}\left(\left(F^{\prime}\right)^{2}+\frac{2 \sin ^{2}(F)}{r^{2}}\right)-\frac{1}{4}\left(\eta^{\prime}\right)^{2}\right]>0, \\
& \frac{\delta^{2} M}{\delta \beta^{2}}>0 \rightarrow \int_{0}^{\infty} r^{2} d r\left(\lambda \eta^{4}+\mu^{2} \eta f_{\pi}(\cos (F)-1)\right)>0 .
\end{aligned}
$$

The first condition does not depend on $e$ neither on $f_{\pi}$. However the second one depends on the value of $\lambda$. This is found in numerical calculations where for very small values of $\lambda$ (and therefore of $m_{\eta}$ ) the solution for $\eta(r)$ becomes so stiffer that it makes the numerical calculations more unreliable and the solutions eventually inexistent for the boundary conditions we consider.

For case (iii) we have the following stability conditions:

$$
\begin{aligned}
& \frac{\partial^{2} M}{\partial \alpha^{2}}>0 \rightarrow \int_{0}^{\infty} r^{2} d r\left\{\frac{4}{f_{\pi}^{2}}\left(2 \sin ^{2}(F) \frac{\left(F^{\prime}\right)^{2}}{r^{2}}+\frac{\sin ^{4}(F)}{r^{4}}\right) \eta^{4}-\frac{\eta^{2}}{4}\left(\left(F^{\prime}\right)^{2}+\frac{2 \sin ^{2}(F)}{r^{2}}\right)-\frac{\left(\eta^{\prime}\right)^{2}}{4}\right\}>0, \\
& \frac{\delta^{2} M}{\delta \beta^{2}}>0 \rightarrow \int_{0}^{\infty} r^{2} d r\left(2 \lambda \eta^{4}+16 e^{2} \eta^{4}\left(\frac{\sin ^{2}(F)\left(F^{\prime}\right)^{2}}{r^{2}}+\frac{\sin ^{4}(F)}{2 r^{2}}\right)-\mu^{2} f_{\pi} \eta(1-\cos (F))\right)>0 .
\end{aligned}
$$

The first of these equations depends on a scale $\left(f_{\pi}\right.$ or $\left.e\right)$ for the stability and the second one depends on the relation between two parameters, $\lambda$ and $e$. This can be seen by means of an usual change of variable such as $r \rightarrow e f_{\pi} r$.

With this analysis we see that the coupling of the scalar field to the fourth order Skyrme term as in prescription (5) is not suitable for the stability of the Skyrmion solution as it was argued before.

\section{$5 \quad$ Summary and Conclusions}

We have investigated the effect of coupling a classical scalar isoscalar field to the usual Skyrmion model as a degree of freedom whose corresponding quantum would be the chiral partner of the pion. 
The dependence of the observables on the values of the scalar field mass and on the fourth order coupling term $e$ were also considered. Smaller values of the $\sigma$ mass favor better agreement for most of the nucleon static properties, except $g_{A}$. Besides the expected solution of small values of the scalar condensate inside the nucleon, we found the possibility of a higher value for the classical field (and also for its mass) inside the nucleon, but for restricted values of the free parameters. This would be equivalent to a further, stronger, chiral symmetry breaking [28, 32]. This feature is not yet well understood and is being investigated [30].

This non trivial solution for the chiral radius (equivalently, for the sigma classical field) may suggest that the chiral symmetry is realized in the Wigner-Weyl mode inside the soliton instead of the Nambu-Goldstone mode, which is expected to hold in the vacuum. The scalar field mass is another free parameter that determines the form of the potential which presents (or not) the spontaneous symmetry breaking. The coupling to the nucleon allows the definition of an effective mass parameter, given by expression (12), which may be even positive inside the nucleon for the solutions shown in the figures while outside (in the vacuum) it becomes negative yielding the "mexican hat"-type potential for $\eta$.

In this work the pion mass term was considered to break explicitely chiral symmetry and to have the more standard form. However this form is not fully determined unless for the requirement of yielding the leading terms of the axial current divergence - PCAC. Furthermore its coupling to the scalar field is not at all uniquely determined and it may be expected to be related to the trace anomaly of QCD [33] and certainly to the chiral SSB. Alternative schemes for that will be shown elsewhere.

As discussed above there is a procedure for obtaining the fourth order stabilizing term from a coupling to the $\rho$ meson field (and also to its axial chiral partner $A_{1}$ ). It is based on the elimination of the vector meson degrees of freedom by performing the infinite mass limit and keeping the KSRF relation [26]: $m_{\rho}^{2}=2 g_{\rho \pi \pi}^{2} f_{\pi}^{2}$. However, we have consistently considered that inside the nucleon $f_{\pi}$ becomes a dynamical degree of freedom whose value may be seemingly reduced. Therefore the limit of large rho mass is not necessarily reliable anymore, thus imposing difficulties in order to relate one picture (with the $\rho$ ) to another (without it). This will be shown in detail elsewhere.

\section{Acknowledgements}

This work was supported by FAPESP, Brazil. The authors thank Dr. M. R. Robilotta for several 
discussions including the idea of prescription (5). I. P. C. also thanks Dr. F. F. da Souza Cruz Filho for several fruitful discussions, which have taken place in the Nuclear Theory Department of the University of Washington, Seattle, U.S.A. This author is thankful to Dr. Larry Wilets for their hospitality. F.L.B. thanks Dr. F.S. Navarra for motivating support and interest in the subject and interesting discussion.

\section{References}

[1] S. Weinberg, Physica A 96, 327 (1979).

[2] T.H.R. Skyrme, Proc. Roy. Soc. Ser. A 260127 (1961).

[3] B.J. Schroers, hep-th/0212076.

[4] G. 't Hooft, Nucl. Phys. B 75, 461 (1974).

[5] E. Witten, Nucl. Phys. B 233, 422, 433, (1983).

[6] G.S. Adkins, in Chiral Solitons, ed. by G.S. Adkins, Add.-Wesley, 1988; O.L. Battistel, Doctoral thesis, Instituto de Física da USP, unpublished, IF-USP, São Paulo, SP, Brazil, 1994.

[7] A.J. Schramm and B. Svetitsky, Phys. Rev. D 62, 114020 (2000).

[8] H. Gomm, P. Jain, R. Johnson, J. Schechter, Phys. Rev. D 33, 3476 (1986).

[9] T. Waindzoch, J. Wambach, Phys. Lett. B 295, 16 (1992).

[10] For example: A. Chodos, R.L. Jaffe, K. Johnson, C.B. Thorn, Phys. Rev. D 10, 2599 (1974).

[11] M.A. Shifman, A.I. Vainshtein, V.I. Zakharov, Nucl. Phys. B 147, 385 (1979); B 147, 448 (1979).

[12] U.G.-Meissner, R. Johnson, N.W. Park, J. Schechter, Phys. Rev. D 371285 (1988).

[13] F.L. Braghin, work presented in Reunião de Trabalho de Física Nuclear no Brasil (Annual Brazilian Meeting on Nuclear Physics), Itatiaia, RJ, Brazil, (1998), without Proceedings..

[14] I. P. Cavalcante, M. R. Robilotta, Phys. Rev. C 63044008 (2001).

[15] M. Gell-Mann and M. Lévy, Nuovo Cimento 16, 705 (1960). 
[16] N.A. Tornqvist and M. Roos, Phys. Rev. Lett. 76, 1575 (1996).

[17] E.M. Aitala et al, Phys. Rev. Lett. 86, 770 (2001) - (hep-ex/0007028).

[18] R.M. Barnett et al, Rev. Mod. Physics 68611 (1996); Review of Particle Physics, Phys. Rev. D 54, 1 (1996).

[19] S. Weinberg, Phys. Rev. Lett. 18, 188 (1967); M.R. Robilotta, classes and lecture notes on "Hadronic Physics", IF-USP, 1991.

[20] F.L. Braghin, M.Sc. thesis, unpublished, IFT-UNESP, São Paulo, SP, Brazil, 1992.

[21] J.F. Donoghue, E. Golowich, B.R. Holstein, Phys. Rev. Lett. 53, 747 (1984).

[22] T.N. Pham, T.N. Truong, Phys. Rev. D 31, 3027 (1985); M. Mashaal, T.N. Pham, T.N. Truong, Phys. Rev. D 34, 3484 (1986).

[23] I. P. Cavalcante, J. Sá Borges, S. Wulck, in Proceedings of XXIII Encontro Nacional de Física de Partículas e Campos (Annual Meeting on Particle Physics and Fields), http://www.sbf1.if.usp.br/eventos/enfpc/xxiii/procs/RES340.pdf (2002); S. Wulck, J. A. Mignaco, in Proceedings of XXII Encontro Nacional de Física de Partículas e Campos, http://www.sbf1.if.usp.br/eventos/enfpc/xxii/procs/trb0161.pdf (2001) (in Portuguese, with one picture).

[24] H.G. Dosch, S. Narison, Phys. Lett. B 184, 78 (1987).

[25] G.H. Derrick, J. Math. Phys. 5, 1252 (1964).

[26] M. Bando, T. Kugo, K. Yamawaki, Phys. Rept. 164, 217 (1988); U.G.-Meissner, Phys. Rept. 161, 213 (1988).

[27] F.L. Braghin, Phys. Rev. D 57, 3548 (1998).

[28] F.L. Braghin, Phys. Rev. D 64, 125001 (2001).

[29] D.O. Riska, nucl-th/9908065.

[30] F.L. Braghin and I.P. Cavalcante, under development; F.L. Braghin, under development.

[31] I. Aitchison, C. Fraser, E. Tudor, J. Zuk, Phys. Lett. B 165, 162 (1985). 
[32] In medium calculation: P.K. Panda and F.L. Braghin, Phys. Rev. C 66 055207, (2002).

[33] S.R. Beane and U. Van Kolck, Phys. Lett. B 328, 137 (1994).

\section{Figure captions}

\section{Figure 1.}

Profile function $F(r)$ for uncoupled skyrmion (solid line) and taking into account the coupling to the condensate with input parameters: $e=4$ and $m_{\epsilon}=980 \mathrm{MeV}$ (dashed line), $e=5$ and $m_{\epsilon}=980 \mathrm{MeV}$ (dotted-dashed line) and $e=4$ with $m_{\epsilon}=560 \mathrm{MeV}$ (dotted line).

\section{Figure 2.}

Condensate $\eta(r)$ in the presence of the soliton for the three cases with coupling of figure 1. 
Figure 1

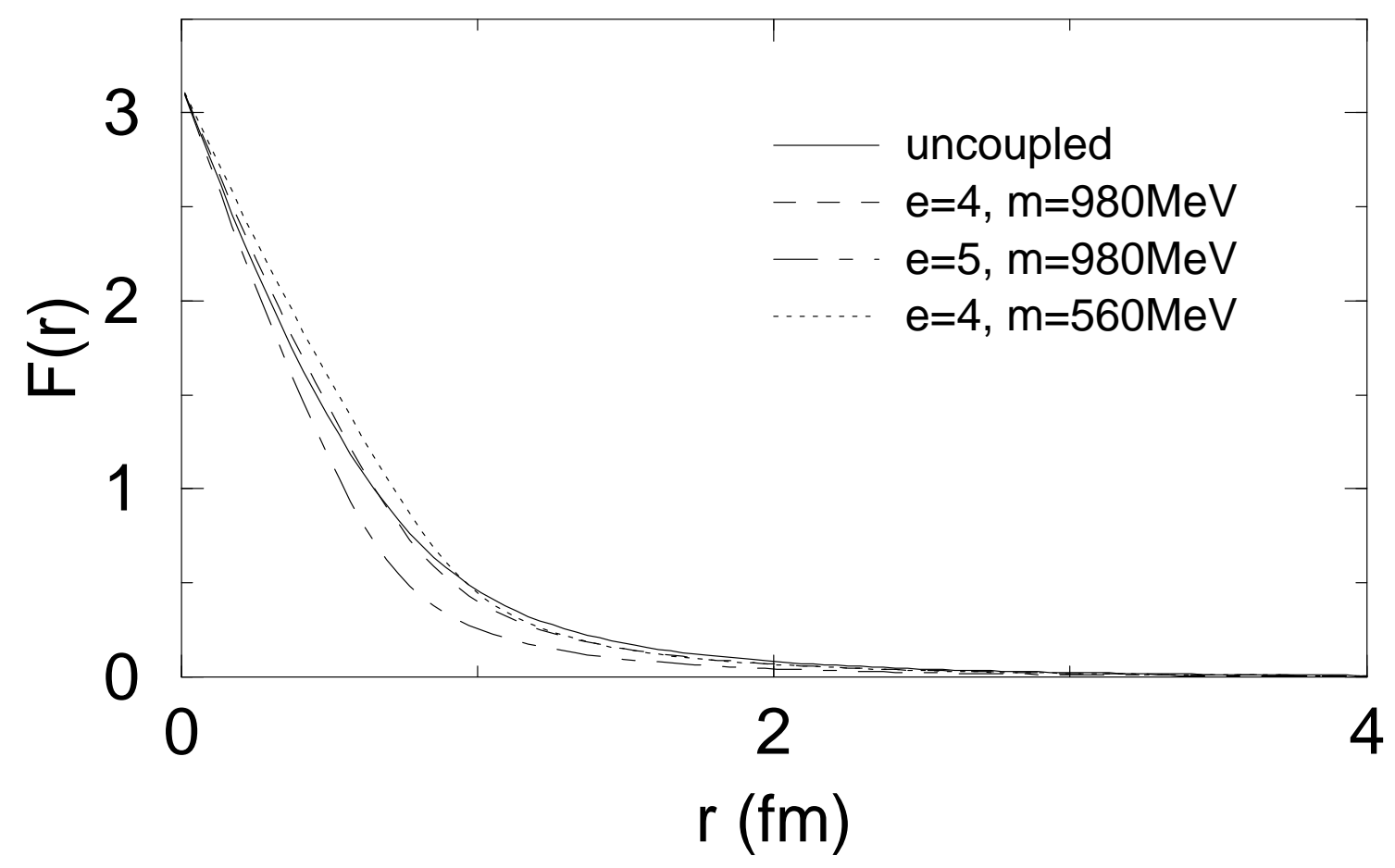

Figure 2

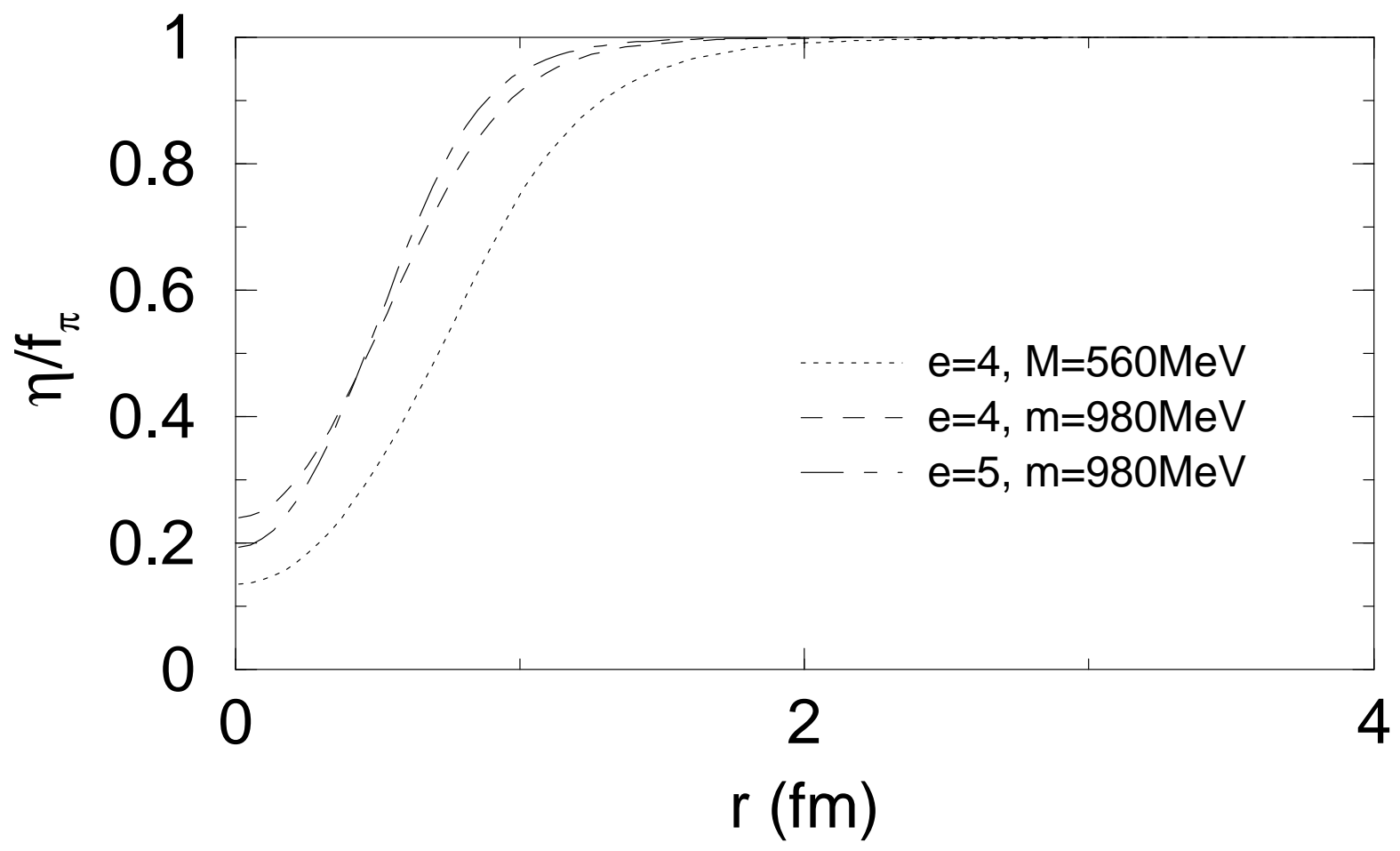


Table 1: Values of the observables calculated for the free skyrmion model and the other three cases discussed in the text (with $f_{\pi}=93 \mathrm{MeV}$ ). $g_{A}$ is calculated in two ways, from the zero momentum nucleon axial form factor as well as from the Goldberger-Treiman relation.

\begin{tabular}{|c|c|c|c|c|c|}
\hline observable & $\begin{array}{c}\text { free } \\
\text { skyrmion }\end{array}$ & $\begin{array}{c}\mathrm{e}=4, \\
m_{\epsilon}=980 \mathrm{MeV}\end{array}$ & $\begin{array}{c}\mathrm{e}=5, \\
m_{\epsilon}=980 \mathrm{MeV}\end{array}$ & $\begin{array}{c}\mathrm{e}=4, \\
m_{\epsilon}=560 \mathrm{MeV}\end{array}$ & experiments \\
\hline$M_{N}(\mathrm{MeV})$ & 1819 & 1578 & 1282 & 1436 & 939 \\
\hline$M_{\Delta}(\mathrm{MeV})$ & 2069 & 1876 & 1830 & 1722 & 1210 \\
\hline$\lambda\left(\mathrm{MeV}^{-1}\right)$ & 166.7 & 198 & 366 & 191 & - \\
\hline$\sigma$-term & 49.6 & 62 & 51 & 54 & $50 \pm 20$ \\
\hline$<r_{E}^{2}>_{B}^{\frac{1}{2}}(\mathrm{fm})$ & .51 & .51 & .43 & .56 & .72 \\
\hline$<r_{E}^{2}>_{V}^{\frac{1}{2}}(\mathrm{fm})$ & .87 & .80 & .71 & .82 & .88 \\
\hline$<r_{M}^{2}>_{B}^{\frac{1}{2}}(\mathrm{fm})$ & .82 & .53 & .44 & .56 & .56 \\
\hline$<r_{M}^{2}>_{V}^{\frac{1}{2}}(\mathrm{fm})$ & .80 & .62 & .55 & .67 & .64 \\
\hline$<r_{E}^{2}>_{p}^{\frac{1}{2}}(\mathrm{fm})$ & .86 & .67 & .59 & .71 & .70 \\
\hline$<r_{E}^{2}>_{n}^{\frac{1}{2}}(\mathrm{fm})$ & -.24 & -.19 & -.16 & -.18 & -.12 \\
\hline$<r_{M}^{2}>_{p}^{\frac{1}{2}}(\mathrm{fm})$ & .66 & .61 & .53 & .63 & $.86 \pm .06$ \\
\hline$<r_{M}^{2}>_{n}^{\frac{1}{2}}(\mathrm{fm})$ & .68 & .64 & .59 & .65 & $.88 \pm .07$ \\
\hline$\mu^{B}$ (magn.) & .34 & .35 & .37 & .36 & .44 \\
\hline$\mu^{V}$ (magn.) & 3.64 & 2.66 & 1.17 & 2.5 & 2.35 \\
\hline$\mu_{p}($ magn. $)$ & 3.97 & 3.01 & 1.54 & 2.9 & 2.79 \\
\hline$\mu_{n}($ magn. $)$ & -3.30 & -2.31 & -.80 & -2.1 & -1.91 \\
\hline$\mu_{\Delta++}(\operatorname{magn})$. & 7.55 & 5.84 & 3.21 & 5.6 & $4.7-6.7$ \\
\hline$\mu_{N \Delta}(\operatorname{magn})$. & 2.66 & 2.24 & 1.21 & 2.32 & 3.3 \\
\hline$g_{A}\left(g_{A}\right.$ GT rel. $)$ & $1.01(1.01)$ & $.76(0.84)$ & $.49(.54)$ & $.78(.83)$ & 1.23 \\
\hline$g_{\pi \pi N}$ & 20.3 & 14.5 & 7.5 & 12.7 & 13.5 \\
\hline$<r_{A}^{2}>^{\frac{1}{2}} \mathrm{fm}$ & .49 & .86 & .85 & .48 & .68 \\
\hline$<r_{\pi}^{2}>^{\frac{1}{2}} \mathrm{fm}$ & .6 & .50 & .37 & .28 & .54 \\
\hline
\end{tabular}

